\title{
Submerged Fermentation of Penicillium paxilli Biosynthesizing Paxilline, a Process Inhibited by Calcium-induced Sporulation
}

\author{
By MICHAEL IBBA, STEPHEN J. C. TAYLOR, \\ CHRISTOPHER M. WEEDON AND PETER G. MANTLE* \\ Department of Biochemistry, Imperial College, London SW7 2AZ, UK
}

(Received 7 April 1987)

\begin{abstract}
A submerged fermentation process for the production of the tremorgenic mycotoxin paxilline by Penicillium paxilli has been developed. The fungus did not sporulate and accumulated paxilline to $1.5 \%(\mathrm{w} / \mathrm{w})$ in freeze-dried cells within $6 \mathrm{~d}$ in a 601 stirred fermenter. Induction of extensive differentiation of conidiophores and profuse sporulation by adding $2 \%(\mathrm{w} / \mathrm{v}) \mathrm{CaCl}_{2} \cdot 2 \mathrm{H}_{2} \mathrm{O}$ to the medium at batching reduced paxilline yield by $97 \%$. Paxilline biosynthesis occurred when the glucose in the medium had been exhausted, implying that carbon catabolite repression may be involved in the biosynthesis of this alkaloid, even when calcium-induced sporulation inhibits or delays the onset of paxilline biosynthesis. Sporulation-induced inhibition of indole-terpenoid alkaloid biosynthesis in $P$. paxilli contrasts with the situation in some other penicillia elaborating indole alkaloids and allows disassociation of aspects of secondary metabolite biosynthesis from growth-associated differentiation, which formerly seemed to be linked.
\end{abstract}

\section{INTRODUCTION}

The biosynthesis of secondary metabolic products of fungi is sometimes associated with specific cellular diffentiation, such as occurs during asexual sporulation, so that, for example, Bu'lock (1975) concluded that secondary metabolism is an aspect of the differentiation which limited growth usually implies. Representatives of two structurally distinct groups of tremorgenic mycotoxins, verruculogen representing the diketopiperazines and the penitrems representing the indole-terpenoids, illustrate this principle, since penicillia which readily elaborate these molecules while growing as sporulating mats in stationary liquid culture apparently produce them in submerged culture only when penicillus differentiation is induced by $\mathrm{Ca}^{2+}$ (Day et al., 1980; Mantle et al., 1984).

Another indole-isoprenoid tremorgen, paxilline, has recently commanded renewed interest since, in addition to being a metabolite of Penicillium paxilli (Cole et al., 1974), it has been shown to be synthesized by the endophytic fungus Acremonium loliae (Weedon \& Mantle, 1987). The intimate association of $A$. loliae with its ryegrass (Lolium perenne) host has been correlated with the occurrence of the indole-terpenoid lolitrems (Gallagher et al., 1984) which cause the important spasmodic neurological disorder of ruminants known as ryegrass staggers. The mechanism of regulation of indole-terpenoid biosynthesis in $A$. loliae, whether concerning paxilline only or also concerning lolitrem $B$ (of which paxilline may be a precursor), is therefore of agricultural importance in the context of the endophyte/host interaction. A formal model of paxilline biosynthesis per se is potentially available in the isolate of $P$. paxilli which has been shown to produce it in stationary liquid cultures (Cole et al., 1974; Cockrum et al., 1979) and has thus been studied to provide some mechanistic understanding as a background against which to explore putative regulatory mechanisms in $\boldsymbol{A}$. loliae. Development of a large-scale submerged process has provided an effective source of paxilline for animal studies since this neurotoxin also occurs in endophyte-infected ryegrass (Weedon \& Mantle, 1987). From the previous studies on developing submerged-culture processes for tremorgenic mycotoxin production (Day et al., 
1980; Mantle et al., 1984) it was expected that calcium-induced sporulation would be a prerequisite for success. In finding otherwise, the present investigation seeks also to identify some of the fundamental mechanisms in the initiation of paxilline biosynthesis and to discuss the wider topic of tremorgenic indole alkaloid production.

\section{METHODS}

Organism. The strain of Penicillium paxilli Bain. used in this study (ATCC 26601) was supplied by J. I. Pitt, CSIRO, Division of Food Research, North Ryde, Australia, where the organism is catalogued as FRR 1900.

Media. The liquid media used were based on Czapek Dox broth supplemented with yeast extract (CDYE broth), $\mathrm{pH} 7 \cdot 3$, containing ( $\mathrm{g} \mathrm{l}^{-1}$ in distilled water): sucrose, $30 ; \mathrm{NaNO}_{3}, 3 ; \mathrm{K}_{2} \mathrm{HPO}_{4}, 1 ; \mathrm{MgSO}_{4} .7 \mathrm{H}_{2} \mathrm{O}, 0.5 ; \mathrm{KCl}, 0.5$; $\mathrm{FeSO}_{4} .7 \mathrm{H}_{2} \mathrm{O}, 0.01$; yeast extract (Difco), 5. Addition of $\mathrm{Ca}^{2+}$, where required, was achieved with $\mathrm{CaCl}_{2} \cdot 2 \mathrm{H}_{2} \mathrm{O}$ $\left(20 \mathrm{~g}^{-1}\right)$.

Inoculum development. Cultures were grown on potato dextrose agar for $7 \mathrm{~d}$ at $27^{\circ} \mathrm{C}$. Sporing mycelium from $15 \mathrm{ml}$ potato dextrose agar was macerated in $100 \mathrm{ml}$ CDYE broth in a baffled $500 \mathrm{ml}$ Erlenmeyer flask and incubated for $24 \mathrm{~h}$ on a rotary shaker ( $200 \mathrm{r}$.p.m.; $10 \mathrm{~cm}$ eccentric throw), then $10 \mathrm{ml} \mathrm{samples} \mathrm{of} \mathrm{this} \mathrm{primary-stage}$ culture were transferred to production-stage (unbaffled) flasks containing $100 \mathrm{ml}$ medium. For pilot-plant fermentations $100 \mathrm{ml}$ of a $24 \mathrm{~h}$ primary-stage culture was transferred to $11 \mathrm{CDYE}$ broth in a $4 \mathrm{l}$ conical flask with side arm and incubated for a further $24 \mathrm{~h}$ at $27^{\circ} \mathrm{C} ; 21$ of secondary-stage inoculum was used to inoculate a 601 stirred fermenter.

Shaken flask fermentations. Four-litre flasks containing 11 CDYE broth were inoculated with $100 \mathrm{ml}$ primarystage culture and incubated for $12 \mathrm{~d}$.

In the first experiment in $500 \mathrm{ml}$ flasks, $100 \mathrm{ml}$ CDYE broth was inoculated with $1 \mathrm{ml} 24 \mathrm{~h}$ primary-stage culture. In the later experiments on delayed addition of $\mathrm{CaCl}_{2}, 500 \mathrm{ml}$ flasks containing $100 \mathrm{ml}$ CDYE broth were inoculated with $10 \mathrm{ml}$ primary-stage culture and supplemented with $10 \mathrm{ml}$ sterile $20 \%(\mathrm{w} / \mathrm{v}) \mathrm{CaCl}_{2}$ solution immediately after inoculation or after 8,15 or $24 \mathrm{~h}$ fermentation, and harvested $10 \mathrm{~d}$ after inoculation.

Stirred fermenter fermentations. The vessels used were stainless steel 601 fermenters of conventional design (Banks et al., 1974). Temperature control was achieved by the automatically regulated flow of cooling water through the fermenter jacket. Culture aeration was provided by sparging air $\left(601 \mathrm{~min}^{-1}\right)$ through a ring sparger into the culture fluid, which was agitated by means of a single disc turbine impeller rotating at 367 r.p.m. Polypropylene glycol antifoam (P 2000) was routinely batched in the medium $\left(0.1 \mathrm{ml} \mathrm{l}^{-1}\right)$. Medium (55 l) was prepared in situ and the $\mathrm{pH}$ value adjusted to 6.0 by addition of $\mathrm{NaOH}$. Medium and fermenter were sterilized at $121^{\circ} \mathrm{C}$ for $20 \mathrm{~min}$ by live-steam injection. Allowance was made for condensate subsequently formed during sterilization bringing the post-sterilization volume to $60 \mathrm{l}$. After inoculation, fermenters were monitored for contamination daily throughout the fermentation by microscopic examination of samples, and by culture at $24^{\circ} \mathrm{C}$ and $37^{\circ} \mathrm{C}$ on media favourable for bacterial or fungal contaminants.

Samples for analysis were taken from the fermenter as follows: post-inoculation, 21 ; at 6,12 and $18 \mathrm{~h}, 11$; thereafter 0.51 samples at intervals.

Analytical procedures. Fermenter samples were filtered through Whatman no. 1 paper and cells or filtrate analysed as appropriate.

(i) Mycelial dry weight. The mycelium from at least $100 \mathrm{ml}$ culture was freeze-dried to constant weight.

(ii) Sporulation. Whole culture was examined microscopically to assess differentiation of sporophores. A few $\mathrm{ml}$ of culture were also coarsely filtered through cotton wool to remove hyphae and the spore content of the broth, suitably diluted where necessary, was measured using a haemocytometer.

(iii) Reducing sugars. The concentration of reducing sugars in the culture filtrate was determined by the procedure of Schoorl (1929). To Fehling's solution $(10 \mathrm{ml})$ was added $10 \mathrm{ml}$ of culture filtrate (diluted as appropriate) and the mixture was boiled for $3 \mathrm{~min}$. After cooling, $10 \mathrm{ml} 2 \mathrm{M}-\mathrm{H}_{2} \mathrm{SO}_{4}$ was added, followed by $5 \mathrm{ml}$ $30 \%(\mathrm{w} / \mathrm{v}) \mathrm{KI}$ solution. The mixture was titrated against $0.025 \mathrm{M}-\mathrm{Na}_{2} \mathrm{~S}_{2} \mathrm{O}_{3}$ solution, using starch indicator, and compared against a standard curve for glucose which was linear over the range $0-0.3 \%$. Total sugars, expressed as free reducing sugars after hydrolysis, were measured as above (although against a standard curve for sucrose), but after boiling $5 \mathrm{ml}$ culture filtrate with $5 \mathrm{ml} 1 \mathrm{M}-\mathrm{HCl}$ and neutralizing, after cooling, with $5 \mathrm{ml} 1 \mathrm{M}-\mathrm{NaOH}$.

(iv) Glucose. Glucose concentration in culture filtrate was determined by a glucose oxidase assay processed automatically using a Beckman Glucose Analyser.

(v) $p H$. The $\mathrm{pH}$ value of samples was measured by a $\mathrm{pH}$ meter standardized against phthalate and phosphate buffers over the range $\mathrm{pH} 4-7$.

(vi) Phosphate. Phosphate in the culture filtrate was measured by a method based on that of Fiske \& Subbarow (1925). The modification substituted 4-amino-3-hydroxy-naphthalene-1-sulphonic acid as a reducing agent in place of hydroquinone.

(vii) $\alpha$-Amino nitrogen. A ninhydrin reagent $\left(0.5 \mathrm{~g}\right.$ ninhydrin, $6 \mathrm{~g} \mathrm{KH}_{2} \mathrm{PO}_{4}, 4 \mathrm{~g} \mathrm{Na}_{2} \mathrm{HPO}_{4}, 0.3 \mathrm{~g}$ fructose, distilled $\mathrm{H}_{2} \mathrm{O}$ to $\left.100 \mathrm{ml}\right)$ and a diluting solution $\left(0 \cdot 2 \mathrm{~g}\right.$ potassium iodate in $60 \mathrm{ml} \mathrm{H}_{2} \mathrm{O}$ and $40 \mathrm{ml} 96 \%, \mathrm{v} / \mathrm{v}$, ethanol) 
was prepared. Culture filtrate $(2 \mathrm{ml})$ and ninhydrin reagent $(1 \mathrm{ml})$ were incubated together at $100^{\circ} \mathrm{C}$ for $16 \mathrm{~min}$ and transferred to another waterbath at $20^{\circ} \mathrm{C}$ for $20 \mathrm{~min}$. Diluting solution $(5 \mathrm{ml})$ was added and the absorbance measured at $540 \mathrm{~nm}$. A standard curve for glycine was linear over the range $0-10 \mathrm{mg} \mathrm{l}^{-1}$.

(viii) Nitrate. Nitrate was estimated in culture filtrate by a method based on that of Nicholas \& Nason (1957). Filtered fermentation broth was diluted 500 -fold and $0.5 \mathrm{ml}$ was mixed with $0.5 \mathrm{ml} 2 \mathrm{M}-\mathrm{NaOH}$ in a $5 \mathrm{ml}$ conical flask. Powdered zinc $(25 \mathrm{mg})$ was added and the flask shaken continuously. After 4,6 and 8 min a $0.2 \mathrm{ml}$ sample was removed and centrifuged (MSE Centaur microcentrifuge) for $30 \mathrm{~s}$ to pellet the zinc and stop the reduction. Then $0.1 \mathrm{ml}$ supernatant was taken from each of the three time-course samples and mixed with $0.9 \mathrm{ml}$ distilled $\mathrm{H}_{2} \mathrm{O}, 0.5 \mathrm{ml} 1 \%$ sulphanilamide in $3 \mathrm{M}-\mathrm{HCl}$, and $0.5 \mathrm{ml} 0.02 \% \mathrm{~N}$-(1-naphthyl)ethylenediamine hydrochloride in $\mathrm{H}_{2} \mathrm{O}$. After 10 min the absorbance was read at $540 \mathrm{~nm}$. From each group of these time-course samples the value of maximum absorbance was taken as indicating maximum generation of nitrite.

(ix) Paxilline. A sample of spores was obtained from the fermenter containing $\mathrm{CaCl}_{2}$, at $48 \mathrm{~h}$. Five litres of culture suspension was poured into five 11 measuring cylinders. After several minutes the pelleted mycelium had largely sedimented, leaving a supernatant suspension of spores which was decanted and centrifuged to form a spore pellet. The pellets were resuspended in a little distilled water and freeze-dried.

Spores prepared as above, and other samples of freeze-dried P. paxilli, were treated with acetone for $24 \mathrm{~h}$. The solvent was filtered off and the cells were re-extracted in acetone for a further $24 \mathrm{~h}$. Combined acetone extracts were taken to dryness under reduced pressure.

Acetone extracts were dissolved in an appropriate volume of $\mathrm{CHCl}_{3}$ and $20 \mu \mathrm{l}$ injected into an HPLC system using a Waters $\mathrm{Z}$-module containing a $\mu$ Bondapak- $\mathrm{NH}_{2}$ cartridge $(8 \mathrm{~mm} \times 10 \mathrm{~cm})$. The mobile phase was dichloromethane/isopropanol $(100: 1, \mathrm{v} / \mathrm{v})$ pumped at $4 \mathrm{ml} \mathrm{min}^{-1}$. Spectrophotometric detection $(281 \mathrm{~nm})$ linked to a chart recorder facilitated quantitation of injected paxilline over the range $0-2 \cdot 5 \mu \mathrm{g}$.

Preparative isolation of paxilline from a 60 l fermentation. At the end of the 601 fermentation without $\mathrm{CaCl}_{2}$, the culture (40 l), together with fermenter washings, was filtered through Whatman no. 50 paper to retain the cells. The filter cake was treated with acetone (10 1) for $65 \mathrm{~h}$, filtered and the mycelium re-extracted in acetone (10 1) for $48 \mathrm{~h}$. Combined acetone extracts were taken to dryness in a rotary evaporator and residual water was removed by freeze-drying. The acetone extract was dissolved in chloroform $(200 \mathrm{ml})$, and silica gel $(100 \mathrm{~g}$, Kieselgel $60,230-$ 400 mesh, Merck) was added. The solvent was evaporated and the remaining brown powder applied to the top of a silica gel column $(1 \mathrm{~kg})$ prepared as a slurry using 41 chloroform/acetone $(15: 1, \mathrm{v} / \mathrm{v})$ and settled for $15 \mathrm{~h}$ in a sintered column $(8 \times 82 \mathrm{~cm})$. The same chloroform/acetone solvent was used for elution under gravity until brown solute components had reached the bottom and 24 samples of $300 \mathrm{ml}$ had been collected. Each fraction was examined by TLC (Camlab polygram SIL G/UV254) in chloroform/acetone (15:1). Spraying with Ehrlich's reagent $(2 \% p$-dimethylaminobenzaldehyde in concentrated $\mathrm{HCl})$ followed by heating with a hairdryer for $1 \mathrm{~min}$ revealed paxilline by its colour, yellow becoming green. Complementary indication of paxilline (Cole et al., 1974) used toluene/ethyl acetate/formic acid $\left(5: 4: 1\right.$, by vol.) as developing solvent and visualization $\left(R_{F} \sim 0.75\right)$ by spraying with $50 \%$ ethanolic $\mathrm{H}_{2} \mathrm{SO}_{4}$ and heating at $100{ }^{\circ} \mathrm{C}$ for $5 \mathrm{~min}$.

Paxilline eluted from the silica gel column in fractions 11-21 inclusive. The solutes from these were combined and resolved by flash chromatography (Still et al., 1978) in a $5 \times 29 \mathrm{~cm}$ column, using $75 \mathrm{~g}$ silica and $400 \mathrm{ml}$ chloroform/acetone (15:1), which was also used for column elution. A series of $35 \mathrm{ml}$ samples was collected and TLC showed that paxilline appeared first in fraction 6, though apparently purest in fractions 13-31, from which paxilline was crystallized $(1.9 \mathrm{~g})$.

\section{RESULTS}

\section{Shaken flask fermentations}

In exploratory $500 \mathrm{ml}$ shaken flask cultures the courses of biomass accumulation and paxilline yields were as represented in Fig. 1. The rate of paxilline biosynthesis declined 2-3 d after maximum biomass accumulation and paxilline then reached a concentration of $\sim 1.6 \%(\mathrm{w} / \mathrm{w})$, which was subsequently sustained. This demonstration, for the first time, of submerged culture production of paxilline in significant yield justified extension of the study to stirred fermenters. Preliminary evidence of an inhibitory effect of $\mathrm{CaCl}_{2}$ on paxilline biosynthesis (Fig. 1) pointed to a need to include study of this aspect also in stirred fermenters.

\section{0 l fermentation in CDYE broth}

Biomass accumulation was complete within $36 \mathrm{~h}$ of inoculating the fermenter (Fig. 2). The mycelium was in the form of branched septate hyphae, some of which were small fragments; others were organized into small granular pellets. No spores were produced and no branching characteristic of the differentiation of penicilli was evident. 


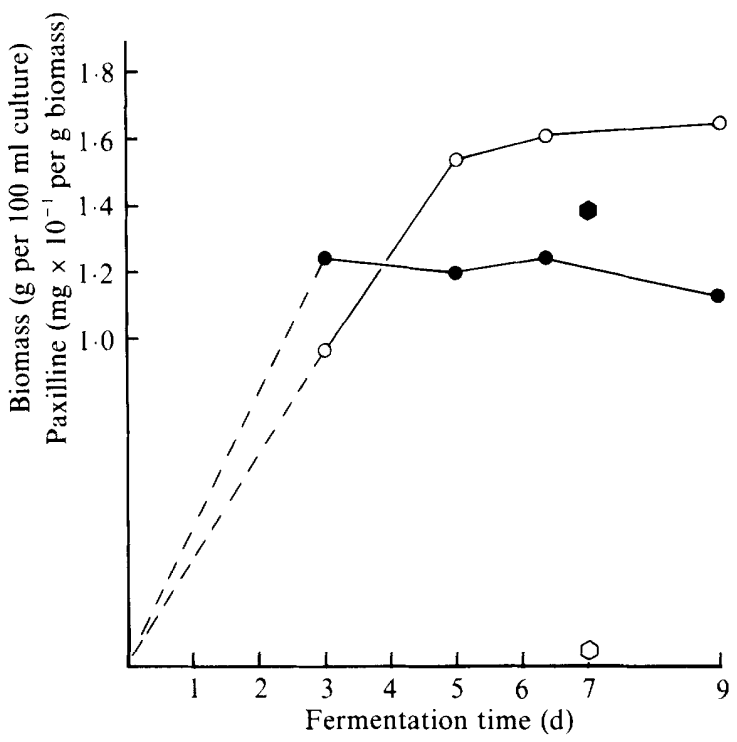

Fig. 1. Progress of $500 \mathrm{ml}$ shaken flask fermentation of $P$. paxilli in CDYE broth. $\bigcirc$, Biomass; $\bigcirc$, paxilline yield. The relative biomass $(\bullet)$ and paxilline yield $(0)$ in a flask supplemented with $\mathrm{CaCl}_{2}$ are also shown.

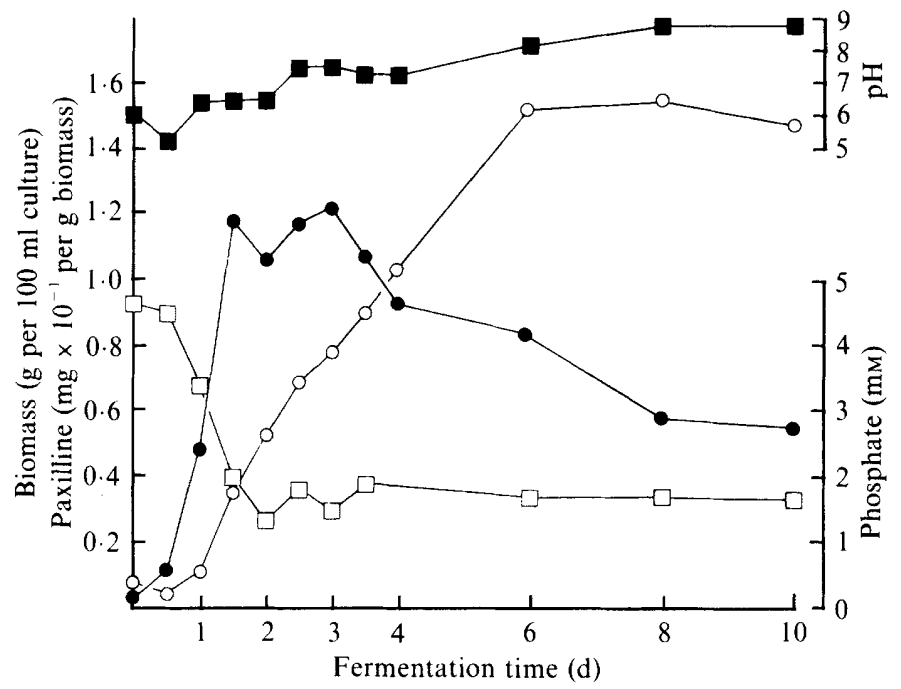

Fig. 2. Progress of stirred 601 fermentation of $P$. paxilli in CDYE broth. $\bigcirc$, Biomass; $\bigcirc$, paxilline yield; $\square$, broth $\mathrm{pH} ; \square$, broth phosphate.

The uptake of phosphate from the medium (Fig. 2) was concomitant with growth during the first $36 \mathrm{~h}$, but was not complete and therefore growth had not been limited at this stage by the availability of this nutrient. Nitrogen sources, batched mainly in the form of nitrate but also including a range of amino acids, provided by the yeast extract, were detected only in low concentration as growth ceased (Fig. 3). In the case of $\alpha$-amino nitrogen this may have represented amino acids and peptides that were not utilizable. The assay of nitrate, while clearly demonstrating considerable uptake during the $36 \mathrm{~h}$ period of biomass accumulation, was insufficiently sensitive to be reliable in determining small concentrations in culture broth. 


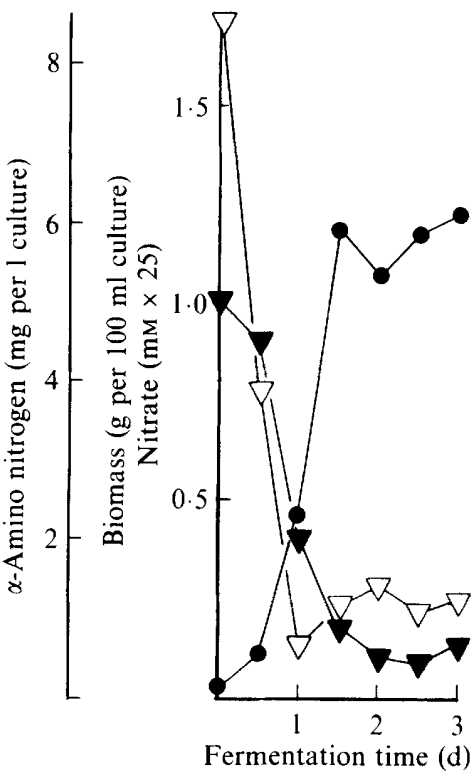

Fig. 3

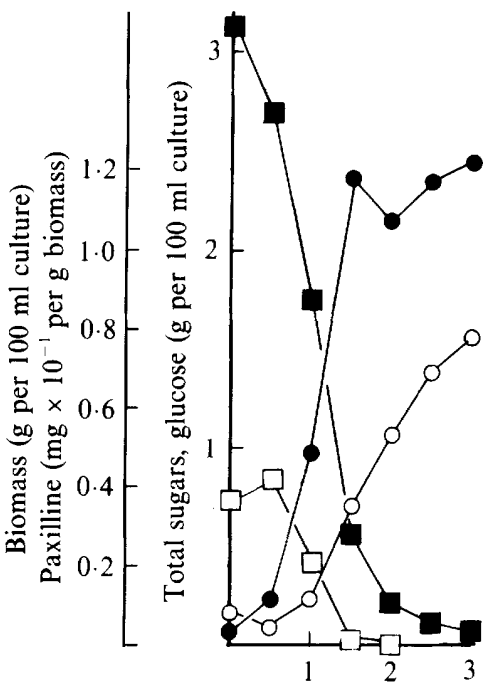

Fermentation time (d)

Fig. 4

Fig. 3. Progress of stirred 601 fermentation of $P$. paxilli in CDYE broth. $\bigcirc$, Biomass; $\nabla$, broth nitrate; $\nabla$, broth $\alpha$-amino nitrogen.

Fig. 4. Progress of stirred 601 fermentation of $P$. paxilli in CDYE broth. $\bigcirc$, Biomass; $\bigcirc$, paxilline yield; $\square$, broth total sugars; $\square$, broth glucose.

However, it was concluded that replicatory growth was probably limited by exhaustion of utilizable nitrogen sources. Approximately half of the carbon source, batched as sucrose, was hydrolysed during sterilization so that approximately $0.75 \mathrm{~g}$ glucose was detected in $100 \mathrm{ml}$ medium in the post-sterilization sample (Fig. 4), and a corresponding amount of fructose would also have been generated. Glucose had disappeared within $36 \mathrm{~h}$ when total reducing sugars, as measured after hydrolysis, were at about $20 \%$ of the amount batched and were probably all in the form of fructose. This had been, in effect, exhausted by $72 \mathrm{~h}$ but seems to have been sufficient to contribute carbon skeletons for the secondary metabolite accumulated after cessation of growth.

Significant paxilline biosynthesis commenced while biomass was still accumulating, but occurred mostly after growth was complete, reaching a maximum value equivalent to $1.5 \%$ of the dry weight of cells by $6 \mathrm{~d}$ (Fig. 2). A small amount of paxilline was detected in the mycelial inoculum, some cells of which had been already 1 or $2 \mathrm{~d}$ in the seed stages of the inoculum development. The measured decrease in paxilline content of mycelium during the first $12 \mathrm{~h}$ in the fermenter is consistent with mycelial proliferation without any biosynthesis of paxilline in the newly formed hyphae.

An initial decline in $\mathrm{pH}$ value (Fig. 2) may reflect some organic acid production in the presence of excess sugar. Thereafter $\mathrm{pH}$ increased to neutrality and became progressively alkaline after $4 \mathrm{~d}$, approximately concomitant with a decrease in biomass and possibly reflecting some hyphal autolysis.

\section{0 l fermentation in CDYE broth supplemented with $2 \% \mathrm{CaCl}_{2}$}

The fermenter batched with additional $\mathrm{CaCl}_{2}$ supported different growth dynamics in which asexual sporulation was a prominent feature.

The seed mycelium inoculated into the fermenter was, as usual, in the form of long, infrequently branched hyphae. By $6 \mathrm{~h}$ the incidence of branching had greatly increased and by 


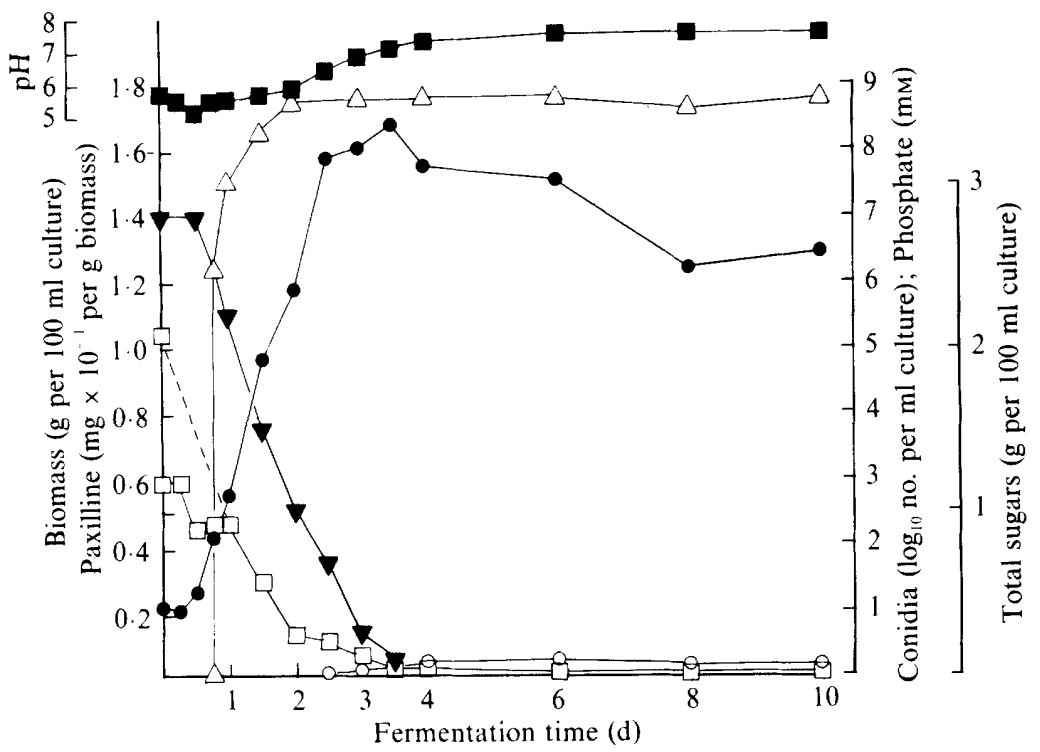

Fig. 5. Progress of stirred $60 \mathrm{l}$ fermentation of $P$. paxilli in $\mathrm{CDYE}$ broth supplemented with $\mathrm{CaCl}_{2}$. Biomass; $\bigcirc$, paxilline yield; $\triangle$, sporulation; $\nabla$, broth total sugars; $\square$, broth phosphate (the graph has been extrapolated back to the batched concentration on account of the unreliability of assays during the first day); $\mathbf{a}$, broth $\mathrm{pH}$.

$12 \mathrm{~h}$ simple penicillus differentiation was evident. At $18 \mathrm{~h}$ many sporophores were seen and at $24 \mathrm{~h}$ they bore chains of conidia; some spores were already free in the broth. At this stage the culture became grey-green, the green becoming more intense over the next $24 \mathrm{~h}$. The broth, yellow up to $24 \mathrm{~h}$, became pink by $36 \mathrm{~h}$ and red by $48 \mathrm{~h}$. A finely divided precipitate of calcium phosphate, which was evident in the fermenter broth before and immediately after inoculation, gradually became associated with the fungal hyphae but by $48 \mathrm{~h}$ had disappeared. Some of the mycelium became aggregated in small pellets which became more obvious as free hyphal fragments autolysed after $6 \mathrm{~d}$.

Biomass achieved a higher value, presumably including a significant amount of the added $\mathrm{Ca}^{2+}$, and it accumulated over a longer period $(60 \mathrm{~h})($ Fig. 5) than in the fermentation without $\mathrm{CaCl}_{2}(36 \mathrm{~h})$. The rate of uptake of reducing sugars was correspondingly decreased (Fig. 6) and the availability of glucose similarly protracted (Fig. 7) but the diauxic utilization of glucose and fructose was clearly evident. The $\alpha$-amino nitrogen and $\mathrm{pH}$ profiles showed a similar pattern but phosphate was more completely taken up from the medium after growth had ceased.

The prominent feature of sporulation, which occurred during the median part of the trophophase, preceded biosynthesis of paxilline. Paxilline biosynthesis occurred only after glucose had been exhausted from the medium, accentuated in Fig. 7 by plotting both the glucose and paxilline dynamics on larger scales. Nevertheless, paxilline biosynthesis amounted to only about $3 \%$ of that in the fermentation without $\mathrm{Ca}^{2+}$. Two other 601 fermentations, similarly supplemented with $\mathrm{Ca}^{2+}$, showed the same trend, giving maximum yields of 0.5 and $4 \%$ of that in the supplemented fermentation, reached at $60 \mathrm{~h}$ and $72 \mathrm{~h}$, respectively.

Surprisingly, the spore fraction $(61 \mathrm{mg})$, separated from 51 of culture ( $60 \mathrm{~g}$ total biomass) at the $48 \mathrm{~h}$ stage, contained $50 \mu \mathrm{g}$ paxilline. The spores were therefore about 50 times richer $(\mathrm{w} / \mathrm{w})$ in paxilline than was the total biomass, of which they were a part, at that time. Since the proportion of the paxilline in the inoculum carry-over attributed to the 51 sample, assuming no catabolism, is $\sim 3 \mathrm{mg}$, the $\sim 1 \mathrm{mg}$ paxilline measured in the 51 sample implies that the $50 \mu \mathrm{g}$ measured in freshly produced $(<30 \mathrm{~h})$ spores is at least most, if not all, of the paxilline synthesized in the fermentation during the first $48 \mathrm{~h}$. 


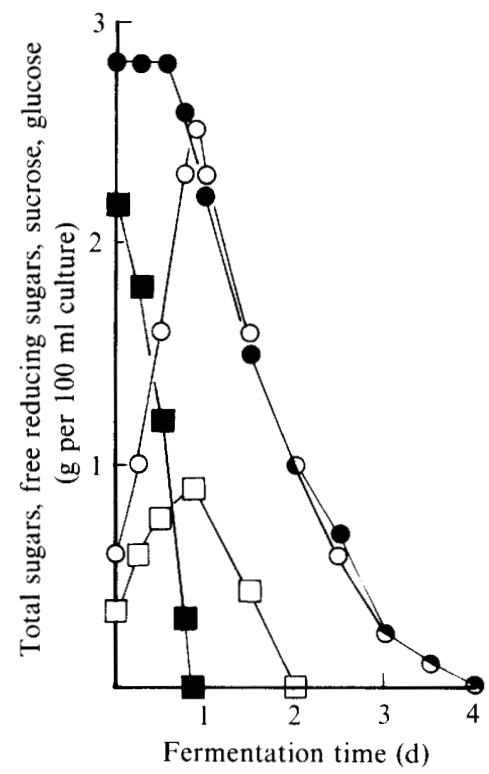

Fig. 6

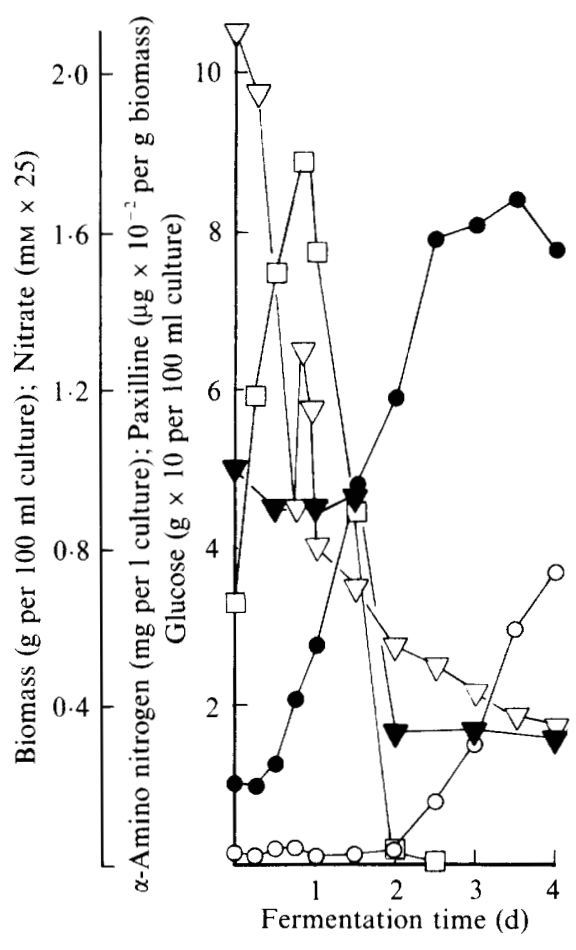

Fig. 7

Fig. 6. Progress of stirred 601 fermentation of $P$. paxilli in CDYE broth supplemented with $\mathrm{CaCl}_{2}$. Broth total sugars; $O$, broth free reducing sugars; $\square$, broth sucrose; $\square$, broth glucose.

Fig. 7. Progress of stirred $60 \mathrm{l}$ fermentation of $P$. paxilli in CDYE broth supplemented with $\mathrm{CaCl}_{2}$ Biomass; $O$, paxilline yield; $\nabla$, broth $\alpha$-amino nitrogen; $\nabla$, broth nitrate; $\square$, broth glucose (scale exaggerated to emphasize temporal relationship with the course of paxilline production).

A shaken flask fermentation of two 11 amounts of $\mathrm{CaCl}_{2}$-supplemented culture in 41 conical flasks inoculated with the same batch of inoculum as the complementary fermenter yielded cells containing $3.8 \mathrm{mg}$ paxilline $\mathrm{g}^{-1}$. This was only about one-quarter of the paxilline yield in the fermenter and may have been a reflection of differences in aeration and agitation in the two types of vessel.

An experiment in $500 \mathrm{ml}$ conical flasks, on the effect of delayed addition of $\mathrm{Ca}^{2+}$, showed that, whether added at the beginning of the fermentation or added 8 or $15 \mathrm{~h}$ later, the fungus yielded $2-3 \times 10^{8}$ spores $\mathrm{ml}^{-1}$ and $\sim 250 \mu \mathrm{g}$ paxilline (g biomass) ${ }^{-1}$. $\mathrm{Ca}^{2+}$ added at $24 \mathrm{~h}$ produced $8 \times 10^{7}$ spores $\mathrm{ml}^{-1}$ and $750 \mu \mathrm{g}$ paxilline $\mathrm{g}^{-1}$. Mycelium in flasks to which $\mathrm{Ca}^{2+}$ was not added did not sporulate and yielded $3.9 \mathrm{mg}$ paxilline $\mathrm{g}^{-1}$.

\section{DISCUSSION}

Comparison of the pattern of growth expressed in submerged fermentations with and without a $\mathrm{CaCl}_{2}$ supplement shows a slower rate of maximum biomass accumulation in the presence of $\mathrm{Ca}^{2+}$. The first dry weight values recorded for the $\mathrm{CaCl}_{2}$-supplemented fermentation were biomass contaminated with a particulate suspension of phosphate, but by $36 \mathrm{~h}$ this contamination was negligible. The apparent lag phase, lasting about $6 \mathrm{~h}$, is therefore accounted for by this artefact. However, the maximum biomass value achieved was much greater in the presence of $\mathrm{Ca}^{2+}(140 \mathrm{~mm})$. This must be attributed to uptake of $\mathrm{Ca}^{2+}$, together with any corresponding adjustment in the intracellular concentration of other ionic species, since Pitt \& 
Poole (1981) showed in a strain of $P$. notatum $[=P$. griseoroseum $(P i t t, 1979)]$ that the rapid uptake of $\mathrm{Ca}^{2+}$ added $(10 \mathrm{mM})$ to submerged cultures to give a mycelial concentration of $12 \mu \mathrm{mol}$ (g dry wt) $)^{-1}$ was matched by a corresponding molar efflux of $\mathrm{Na}^{+}$. Mycelial $\mathrm{Na}^{+}$concentrations decreased from the normal value of approximately $35 \mu \mathrm{mol}$ (g dry wt) ${ }^{-1}$ to about $25 \mu \mathrm{mol}$ (g dry $w t)^{-1}$, thus effecting a nett gain in the biomass equivalent to the difference between the relative atomic masses of $\mathrm{Ca}$ and $\mathrm{Na}$. Intracellular $\mathrm{Mg}^{2+}$ and $\mathrm{K}^{+}$were not affected. Furthermore, the associated nett increase in cations may require additional uptake of anions.

The losses of biomass (per unit volume of culture) after the trophophase are probably due to respiration of assimilated carbohydrate, hyphal autolysis and/or biomass deposition on fermenter walls as the working volume decreased with sampling and evaporation. The different morphologies in the sporing and filamentous cultures will have caused variation in the relative contributions of these three factors.

Discussion of the present fermentations in relation to the series of studies by D. Pitt and coworkers on conidiation in submerged fermentations of $P$. griseoroseum and $P$. cyclopium seems appropriate. In spite of the approximately 15 -fold greater amount of $\mathrm{Ca}^{2+}$ added in the present studies with $P$. paxilli and the use of a two-stage fermentation process involving a more nutritious (although undefined) medium including yeast extract there are many features in common; for example, temperature and aeration conditions were similar to those used by Pitt \& Poole (1981). The present use of a relatively large addition of $\mathrm{CaCl}_{2}$ was simply repetition of the method found advantageous in effecting efficient submerged sporulation and associated maximum production of the characteristic tremorgenic mycotoxins of $P$. simplicissimum (Day et al., 1980), P. raistrickii (Mantle \& Wertheim, 1982) and $P$. janczewskii (Mantle et al., 1984). Pitt \& Poole (1981) and Ugalde \& Pitt (1983) found that calcium-induced conidiation was associated with a diminution in biomass accumulation, particularly in $P$. cyclopium. In the present studies a similar general effect on growth rate was evident but the difference was eventually obscured and then reversed by the overall greater yield of biomass in the presence of $\mathrm{Ca}^{2+}$.

The replicatory component of biomass reflects sugar metabolism. Thus, since Pitt et al. (1983) concluded that the mode of sucrose utilization was similar whether or not calcium-induced conidiation occurred, the present findings of the same rapid inversion of sucrose and a loosely diauxic utilization of the released glucose and fructose is indicative, in each case, of patterns of general metabolic activity in common. The slower uptake of monosaccharides is clearly evident in the sporing fermentation, as is nitrogen source uptake. The two fermentations differed somewhat in the disaccharide/monosaccharide composition at inoculation. In the absence of $\mathrm{CaCl}_{2}$ there was approximately $50 \%$ hydrolysis of the batched sucrose during sterilization. With $\mathrm{CaCl}_{2}$ less than $20 \%$ hydrolysis occurred, but thereafter invertase transformed all remaining sucrose within the first $24 \mathrm{~h}$ of fermentation. Pitt \& Poole (1981) implied that invertase was not a constitutive enzyme and that it disappeared from culture filtrates after sucrose inversion was complete. Thus in the present fermentation with $\mathrm{CaCl}_{2}$ the sudden temporary rise in the concentration of $\alpha$-amino nitrogen associated with the disappearance of sucrose could reflect the products of autolysis of the considerable amount of induced invertase necessary to have hydrolysed more than $1 \mathrm{~kg}$ sucrose in the fermenter.

Gluconate, which Pitt et al. (1983) found accumulating in $P$. griseoroseum grown on glucose without $\mathrm{Ca}^{2+}$, but which was negligible in calcium-induced sporulation, was probably not a significant metabolite in the present $P$. paxilli fermentations. The small decrease in $\mathrm{pH}$ value, occurring in both $P$. paxilli fermentations during the first $12 \mathrm{~h}$, might be attributable to organic acid production before the maximum respiratory demands were imposed on dissolved oxygen. An instance in which organic acid, probably gluconate, is obviously produced is evident during calcium-induced sporulation by $P$. simplicissimum in the trophophase before it then accumulates verruculogen (Day \& Mantle, 1985); a decrease of 1.5 units occurred during the first $48 \mathrm{~h}$, coincident with sugar uptake.

The present studies have demonstrated submerged culture production of paxilline for the first time. The maximum yield of paxilline in the fermenter without $\mathrm{CaCl}_{2}$ compares very favourably with that achieved for penitrems in similar vessels (Mantle et al., 1984). Paxilline was produced more quickly and in greater amount that in a 41 shaken flask, although in a similar yield and 
with dynamics similar to that in $500 \mathrm{ml}$ shaken flasks. Thus no particular constraint on scale-up was apparent. However, the amount of paxilline measured is only the mathematical product of anabolic and catabolic processes; the extent to which paxilline is turned over during the idiophase is unknown. Indeed, since paxilline is only known to be produced by one isolate of Penicillium, the question arises as to whether, in other fungi, it is actually an intermediate in the biosynthesis of some other indole-terpenoid metabolite which fails to accumulate in this organism owing to a defective enzymic step beyond paxilline. In this case typical regulatory mechanisms might not operate properly. Paxilline may even form part of a metabolic grid of indole-terpenoid secondary metabolites such as has been shown in P. griseofulvum for a group of tetraketide metabolites (Bu'Lock, 1975).

The poor yield of paxilline when $P$. paxilli is induced to sporulate by $\mathrm{CaCl}_{2}$ may be due either to diminished biosynthesis or to increased metabolic transformation of paxilline to another product. If diminished biosynthesis is responsible, the finding of most of the paxilline in the spores not only indicates that it is not just the product of enzymes already synthesized in the filamentous inoculum. It also suggests that it is not the actual process of sporulation which inhibits paxilline biosynthesis but rather some changes in the hyphae consequent on their differentiation into spore-forming structures. If increased metabolic transformation is occurring it may be an oxidative process. The expected higher dissolved oxygen content of the broth, resulting from slower sugar uptake and the pelleted sporulating culture's rheological properties being more favourable to oxygen transfer, may promote oxidative catabolism of paxilline. However, there were no qualitative differences between chromotograms of extracts of mycelia grown with or without $\mathrm{CaCl}_{2}$.

In both vegetative and sporulating fermentations the first appearance of paxilline in the cells coincides closely with completion of glucose uptake, whether this occurs early in the second day of solely vegetative fermentation or at the end of the second day in a sporulating culture. A simple conclusion is that regulation of paxilline biosynthesis, at least with respect to the last step, involves release from carbon catabolite repression or inhibition, a classical example of which is the penicillin fermentation.

In the present studies the partial precipitation of calcium phosphate after sterilization and its subsequent gradual dissolution would provide a continuous supply of $\mathrm{Ca}^{2+}$ during the period of sporophore differentiation. It is remarkable that, within only a few hours of formation, spores contained paxilline, while no nett increase in paxilline content of biomass was yet apparent. This apparent incongruity could be rationalized by regarding spores as no different from the hyphae subtending them, in terms of secondary biosynthetic potential. It is possible that $\mathrm{Ca}^{2+}$ taken up into the mycelium is not distributed evenly to spores produced several hours later and that, therefore, their secondary biosynthetic potential is less affected than is that of hyphae.

It is notable also that paxilline seems only to be formed in spores after they have been released into suspension, i.e. after $48 \mathrm{~h}$ into the batch fermentation. Ugalde \& Pitt (1986) showed that as little as 30 s exposure of $P$. cyclopium to $9 \mathrm{~mm}^{-\mathrm{Ca}^{2+}}$ stimulated subsequent differentiation of conidiophores as efficiently as if the fungus was left in the $\mathrm{Ca}^{2+}$ environment. The $\mathrm{Ca}^{2+}$ uptake was biphasic, initial binding to hyphae being followed by active transport of the cation to the subcellular sites described earlier (Ugalde \& Pitt, 1984). However, the study did not extend to include redistribution into spores. Thus these findings may allow discrimination between the effect of calcium on sporulation and its adverse effect on paxilline biosynthesis. The extent to which bound calcium is subsequently absorbed, or absorbed calcium located on membranes or in organelles is transported to new domains in new cells, is not clear. Whether or not spores show the same dynamics as vegetative hyphae with respect to calcium binding and uptake is also unknown.

Paxilline was the only prominent secondary metabolite extracted from the mycelium with organic solvents. However, at about the $24 \mathrm{~h}$ stage the sporulating mycelia developed a green pigment and at about $36 \mathrm{~h}$ a red water-soluble pigment was evident in the broth. This implied anthraquinone biosynthesis suggests some analogy with the gibberellin fermentation (Bu'Lock et al., 1974), in which differential biosynthetic demand on acetate is displayed, a polyketide metabolite (bikaverin) being formed first and the terpenoid gibberellin somewhat later. 
The present findings with $P$. paxilli add a further permutation of apparently contradictory instances concerning the importance of sporulation in submerged culture for the production of tremorgenic mycotoxins. The fact that these compounds have potent tremorgenic activity in animals is probably irrelevant; they simply represent a fairly well explored group of secondary metabolites. Thus $P$. simplicissimum produces the diketopiperazine verruculogen in submerged culture only if induced to sporulate by $\mathrm{CaCl}_{2}$ (Day et al., 1980), but persistently vegetative mycelium of $P$. raistrickii will produce the same secondary metabolite, in at least as good yield, in the presence of $2 \% \mathrm{CaCl}_{2}$ (Mantle \& Wertheim, 1982). Within the indole-terpenoid group of tremorgens, penitrems are produced in high yield in submerged culture only by a strain of $P$. janczewskii given $\mathrm{CaCl}_{2}$ (Mantle et al., 1984); other penicillia producing penitrems in stationary liquid culture fail to elaborate more than a trace of penitrems. Conversely, $P$. paxilli producing paxilline, the structure of which is virtually identical to a large part of the penitrem molecule, does so in submerged culture without sporing and production is severely impaired if the fungus is forced to sporulate.

Exploring a hypothesis that secondary metabolites might act as morphogens in conidiogenesis by $P$. urticae $[=P$. griseofulvum (Pitt, 1979)], Sekiguchi \& Gaucher (1977) studied the fungus in submerged culture during sporulation induced by $\mathrm{Ca}^{2+}$. Sporulation occurred during the trophophase and the polyketide patulin was produced only after sporulation, negating a role in differentiation. However, Sekiguchi \& Gaucher (1977) also recorded a 75\% reduction in the yield of patulin when the fungus was forced to sporulate by addition of $\mathrm{CaCl}_{2} .2 \mathrm{H}_{2} \mathrm{O}(1 \%$, w/v) to the medium at batching. This is therefore qualitatively similar to the present findings with $P$. paxilli.

Thus, while Bu'Lock's (1975) perceived link between secondary metabolism and differentiation may well be valid in certain fungi, the process of sporulation in penicillia, which involves the most complex asexual differentiation that they can achieve, does not seem to be very closely connected with the biosynthesis of secondary metabolites. Nevertheless, calcium-induced conidiogenesis in submerged culture does seem to have consequences for the operation of secondary biosynthetic pathways but it is not possible to recognize a consistent pattern of expression. It should be noted that Bu'Lock's conclusion assumed that differentiation is closely associated with growth limitation by a nutrient becoming scarce. Submerged calcium-induced sporulation by penicillia is very much a mid-trophophase event in its fullest expression of large numbers of spores released into suspension. Moreover, the first evidence of the divergent morphology which is a prelude to sporulation is seen only a few hours into a batch fermentation, when all nutrients are abundant. Thus the dynamics of submerged culture sporulation in penicillia may place these rather prominent differentiations outside the scope of Bu'Lock's conclusion.

In any case, the penicillia exhibit such a diverse array of secondary metabolites that further study of the neglected area of biosynthetic regulation in the context of growth dynamics will assist in revealing the extent of commonality of underlying, and perhaps exploitable, principles.

\section{REFERENCES}

Banks, G. T., Mantle, P. G. \& Szczyrbak, C. A. (1974). Large-scale production of clavine alkaloids by Claviceps fusiformis. Journal of General Microbiology 82, 345-361.

Bu'Lock, J. D. (1975). Secondary metabolism in fungi and its relationship to growth and development. In The Filamentous Fungi, I, pp. 35-58. Edited by J. E. Smith \& D. R. Berry. London: Edward Arnold.

Bu'LoCK, J. D., Detroy, R. W., HostaleK, Z. \& MUNIM-AL-ShaKaRCHI, A. (1974). Regulation of secondary biosynthesis in Gibberella fujikuroi. Transactions of the British Mycological Society 62, 377-389.

Cockrum, P. A., Culvenor, C. C. J., Edgar, J. A. \& PAYNE, A. L. (1979). Chemically different tremor- genic mycotoxins in isolates of Penicillium paxilli from Australia and North America. Journal of Natural Products 42, 534-536.

Cole, R. J., Kirksey, J. W. \& Wells, J. M. (1974). A new tremorgenic metabolite from Penicillium paxilli. Canadian Journal of Microbiology 20, 1159-1162.

Day, J. B. \& Mantle, P. G. (1985). Large-scale and $\mathrm{pH}$-controlled fermentation production of the tremorgenic mycotoxin verruculogen. Biotechnology and Bioengineering 27, 1586-1590.

Day, J. B., Mantle, P. G. \& Shaw, B. I. (1980). Production of verruculogen by Penicillium estinogenum in stirred fermenters. Journal of General Microbiology 117, 405-410. 
Fiske, C. H. \& SUbBaRow, Y. (1925). The colorimetric determination of phosphorus. Journal of Biological Chemistry 66, 375-400.

Gallagher, R. T., Hawkes, A. D., Steyn, P. S. \& VLEGGAAR, R. (1984). Tremorgenic neurotoxins from perennial ryegrass causing ryegrass staggers of livestock: structure elucidation of lolitrem B. Journal of the Chemical Society Chemical Communications, 614-616.

Mantle, P. G. \& Wertheim, J. S. (1982). Production of verruculogen during growth of Penicillium raistrickii. Transactions of the British Mycological Society 79, 348-350.

Mantle, P. G., Laws, I., Tan, M. J. L. \& Tizard, M. (1984). A novel process for the production of penitrem mycotoxins by submerged fermentation of Penicillium nigricans. Journal of General Microbiology 130, 1293-1298.

Nicholas, D. J. D. \& Nason, A. (1957). Determination of nitrate and nitrite. Methods in Enzymology 3, 981-984.

PITT, J. I. (1979). The Genus Penicillium and its Teleomorphic States Eupenicillium and Talaromyces. London: Academic Press.

PitT, D. \& Poole, P. C. (1981). Calcium-induced conidiation in Penicillium notatum in submerged culture. Transactions of the British Mycological Society 76, 219-230.

Pitt, D., Mosley, M. J. \& Barnes, J. C. (1983).
Glucose oxidase activity and gluconate production during calcium-induced conidiation of Penicillium notatum in submerged culture. Transactions of the British Mycological Society 81, 21-27.

SCHOORL, N. (1929). Sugar titration, Chemische Weekblad 26, 130-134.

Sekiguchi, J. \& Gaucher, G. M. (1977). Conidiogenesis and secondary metabolism in Penicillium urticae. Applied and Environmental Microbiology 33, 147-158.

Still, W. C., KaHn, M. \& Mitra, A. (1978). Rapid chromatographic technique for preparative separations with moderate resolution. Journal of Organic Chemistry 43, 2923-2925.

Ugalde, U. \& PItT, D. (1983). Morphology and calcium-induced conidiation of Penicillium cyclopium in submerged culture. Transactions of the British Mycological Society 80, 319-325.

Ugalde, U. \& PITT, D. (1984). Subcellular sites of calcium accumulation and relationships with conidiation in Penicillium cyclopium. Transactions of the British Mycological Society 83, 547-555.

Ugalde, U. \& PITT, D. (1986). Calcium uptake kinetics in relation to conidiation in submerged cultures of Penicillium cyclopium. Transactions of the British Mycological Society 87, 199-203.

Weedon, C. M. \& Mantle, P. G. (1987). Paxilline biosynthesis by Acremonium loliae; a step towards defining the origin of lolitrem neurotoxins. Phytochemistry 26, 969-971. 